\title{
The Exploration of the Topical Progression Patterns in English Discourse Analysis
}

\author{
Jian Shi \\ College of Humanities and Foreign Languages, Xi'an University of Science and Technology, China
}

\begin{abstract}
On the basis of a brief literature review about the major topical progression and thematic progression theory models proposed by the scholars at home and overseas, the present article incorporates the TP modal of Converse Linear Progression maintained by Hu Yinglin into the four types of TSP advocated by Simpson. Meanwhile, the paper attempts to make the addition of the Extended Converse Linear Progression to analyze the topical progression in the available popular English narrative discourse "a private conversation" which achieves coherence and finally points out the limitations of thus doing with one text in expectancy to testify the hypothesized pattern within more various genres of discourses.
\end{abstract}

Index Terms - discourse analysis, thematic progression, topical progression extended converse linear progression modal

\section{A Brief Literature Review of Topical Progression And Thematic Progression Studies}

In order to be able to understand fully the coherence and cohesion achieved within a paragraph or text, the different predecessors in the area of discourse analysis made good use of the quite similar but somewhat different theoretical models. Danes (1974) held that a textual information construction was decided by the thematic progression and the characteristics of thematic progression determined the kinds of textual genres. Since then, many linguists began to research theme and thematic progression models. According to Qi Wang \& Xiaotang Cheng (2004), Danes (1974), and Fries $(1983,1995)$ have induced such four fundamental TP models as Simple Linear TP or chain model, TP with a continuous Theme ,TP with derived Themes and the exposition of a split Rheme. Michael McCarthy (1991) concluded the pattern of TP with derived rheme via analyzing the postcard discourse. Shenghuan Xu $(1982,1985)$ advanced two brand-new TP patterns: TP with the same rheme and crossed pattern which are totally distinct from those put forward by Danes, Fries and Michael McCarthy. Based on the work done by the previous linguists, Yan Huang (1985) summarized seven models of TP: the same theme pattern, simple linear TP, the same rheme pattern, crossed pattern, parallel progression pattern, the rheme of the $1^{\text {st }}$ clause becoming the theme of the following clauses, and there being no clear relation between or among the theme and rheme of the clauses in a discourse. Recent years has witnessed some further studies about theme and rheme. Yan Liu (2010) commented on the basic three topical progression patterns: parallel progression, sequential progression and extended parallel progression by disclosing their advantages and disadvantages respectively and maintained the extended parallel progression was superior to the other two. Bing Li (2012) conducted an experimental study of coherence of argumentative writings from the perspectives of genre theory and thematic progression theory. And still Mei Chen (2005), Guoqing Li (2003), Lan Xiang (2002) etc. among many others carried out some practical studies to relate the TP modals with the teaching of English reading, writing and translating.

In this paper, the author tentatively makes the comparison between TSP which was proposed by Lautimatti in 1987, then developed by Simposon in 2000 and the main patterns of thematic progression modals in English discourse explicated by Yinglin $\mathrm{Hu}$ in 2006, hoping to find out their own merits and demerits in an expectation to extract their advantageous thinking modes by combining them to analyze the topical progression patterns of the English discourses.

A. The Models of TSP

The theme-rheme theory is the basis of the systematic-functional linguistic approach. Halliday(1985) takes the theme as "the point of departure of the message" and says that it is developed in the rheme which is "the remainder of the message" within the sentence. The theme can be identified as that element which comes first position in the clause. The thematic patterning is called "thematic structure" (Halliday, 1985) or "thematic progression (TP)" (Danes, 1974) or "method of development" (Fries, 1983; Martin, 1992). The topical structure analysis (TSP) refers to the analyzing of coherence derived by examining the internal topical structure of the paragraph as reflected by the repetition of key words and phrases. Lautimatti(1987) proposed three types of topical structure analysis, namely Parallel Progression(PP two consecutive clauses with the same topical subject), Sequential Progression(SP the comment element of a clause becoming the topic element of the consecutive clause) and the Extended Parallel Progression(EPP a topical subject occurs in two clauses that are not consecutive). Simpson (2000) suggested that there should be the addition of the fourth category---Extended Sequential Progression (ESP, the comment element of a clause becoming the topic element of the discontinuous clause). The extended sequential progression can be regarded as a strategy to link ideas together across a distance in a paragraph. In the TSP model, both Lautimatti and Simpson employ three basic sentence elements which 
play important roles in analyzing the thematic progression. They identified the initial sentence element (ISE), which is what comes first in the sentence. This may be the subject of the sentence, an introductory phrase or clause; the second element is the mood subject (MS) or the grammatical subject of the sentence. And the third element is the topical subject (TS), which may or may not the mood subject. The three TSP modals by Lautimatti and the newly-added modal by Simpson can be diagrammed as follows (cited from Huisong Zhang (2006)):
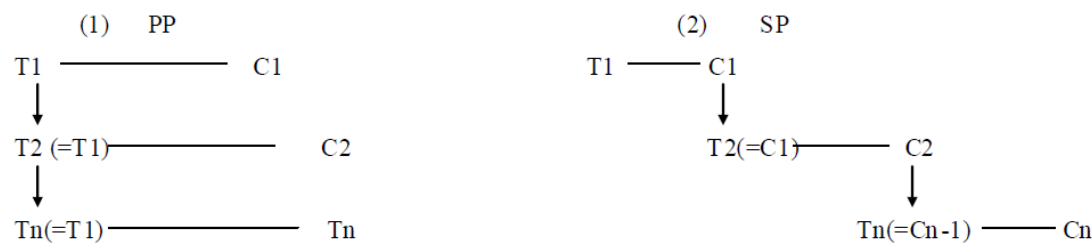

(3) $\quad \mathrm{EPP}$

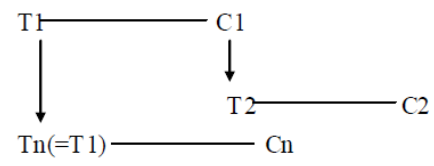

(4) ESP

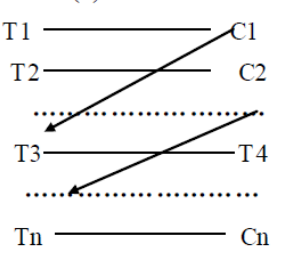

\section{B. Main Patterns of TP Maintained By Yinglin $\mathrm{Hu}$}

From the viewpoint of Yinglin $\mathrm{Hu}$, there just generally lies the parallelism, a kind of rhetorical device, as a kind of link of ideas within a paragraph or discourse.

And he categorizes the main patterns of thematic progression in English as the following four types: Simple Linear Progression (SLP, the rheme in the previous sentence becomes the theme of the next sentence), Constant Theme Progression (CTP, the theme is identical in a sequence of sentences), Constant Rheme Progression (CRP, the rheme is identical in a sequence of sentences) and the Converse Linear Progression (CLP, each theme becomes the rheme of the next sentence). They are diagramed as follows:
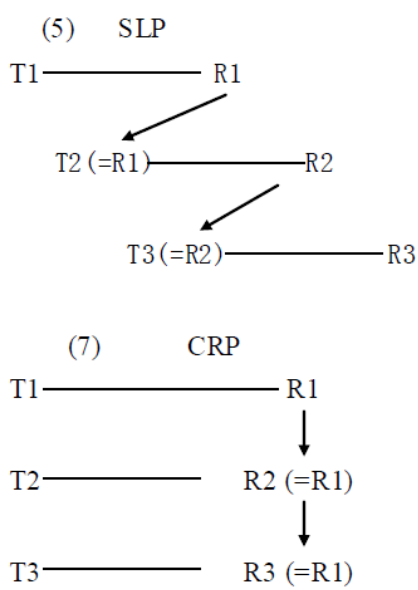

(6) CTP
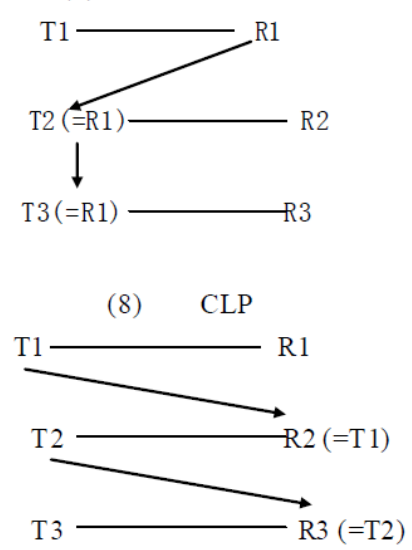

\section{Comparison between TSP and HU's Patterns of TP}

In the Models of TSP, parallelism is dichotomously divided into two subcategories: the parallelism progression and the extended parallelism progression, whereas HU only vaguely put forward the parallelism. Apparently, TSP model is much more concrete and conveniently operationalized.

The four types of thematic progression embraced in the main patterns of Hu deserve respective concern and treatment. The Constant Theme Progression and the Constant Rheme Progression are very formal, much too fixed and metaphysically, which are merely applicable to analyze certain genres of discourse. The Simple Linear Progression is rather identical with the Sequential Progression in TSP, the rheme of the clause changes into the theme of the consecutive clause. What the TSP model ignores here is the opposite phenomenon that the theme of the previous clause becomes the rheme of the following clause. The Converse Linear Progression put forward by Hu fills the gap, which is worthy of our attention and should be integrated into the model of TSP.

Topic structure progression and thematic progression are both concerned about the organizing mode of a paragraph or discourse, but they are also different to some extent. According to Xuanwei Peng (2005), theme is the starting point and 
foundation of the information expressed in a clause and is the first element of relevant information. Huisong Zhang (2005) believed that TSP and TP possessed the common purpose and similar progression models, but differed in their basic analyzing units and priorities. Theme is a position-oriented grammatical formal category while topic belongs to the semantic one. Although TSP and TP show differentiated priorities in the developing methods of texts, they share some similarities in the spreading patterns.

\section{EMPIRICAL STUDY--APPliCATION OF THE Two THEORY MODELS IN PRACTICAL TeXTS}

In the above section of the article, the author expounds two kinds of theory models as regards the modes of development within a paragraph or discourse, making a thorough comparison between them so as to be aware of their individual strengths and shortcomings. It is obvious that the TSP model is a lot advantageous than the patterns held by $\mathrm{Hu}$ in discomposing the unity of discourse development. Hence, the author personally adopts a strong preference for the model of topical structure analysis of Lautimatti and Simpson, suggesting the combination of the TSP model and part of the patterns of thematic progression of Hu. To put it simply, the Converse Linear Progression in the Hu's pattern should be incorporated into the TSP model to analyze the various English Paragraphs and discourses.

\section{A. The Thematic Progression in an Expository Text}

The following paragraph is selected for the discussion of applying the different models of thematic progressions. Italics are used for ISE, underlining is employed to signal mood subject (grammatical subject of the verb), and bold face is for the topical subject.

(1).Every people has its own special words and expression that become part of the language. (2). One such American expression is "eager beaver". (3). An "eager beaver" is a person who is always willing and excited to do what is expected of him. (4). The expression comes from the name of a hard working animal ---the beavers. (5).

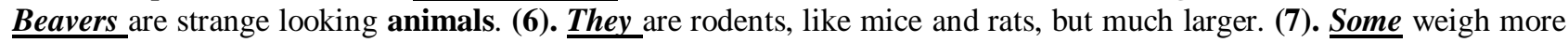
than 25 kilograms. (8). Beavers have a large, black, flat tail to help them swim. (9). They also have thick, brown hair of fur to keep them warm in cold water and they have front teeth sharp enough to cut down large trees. (10). Beavers spend a lot of time in the water building dams to create little lakes or ponds. (11). They work hard to cut down trees, remove branches and put them together with mud to make dams. (12). Few other animals are so hard-working. (13). Historians say the beaver had an important part in the settlement of North America. (14). There were hundreds of millions of beavers when European settlers first arrived. (15). The settlers put great value on the fur of the beavers. (16). In fact, for 200 years or more, beaver was the most valuable fur in North America. (17).Most of the beavers were trapped or killed. (18). For a time, they were in danger of disappearing completely. (19). But laws were passed to protect the beaver. (20). And today the population is rising. (21). In fact, wild beavers are building dams in a national park only 50 kilometers from Washington. (22). And like the animal, the expression "eager-beaver" is in no danger of dying out.

Parallel Progression: In the clause number (5), the noun phrase "Beavers" functions as the ISE, the mood subject and the topical subject. In the next independent clause number (6), the three clause elements coincide in the pronoun "they". Here the word "they" in clause (6) refers to the same topic as the noun phrase "Beavers" from the clause (5). Thereby, this is an example of parallel progression. Similarly, the noun "Beavers" in clause number (8), the pronoun "They" in clause number (9), the noun "Beavers" in clause number (10) and the pronoun "They" in clause number (11) commonly share the three clause elements and co-refers to the same entity and completely illustrate the models of parallel progression.

Extended Parallel Progression: As mentioned above, the relationship between clause (5) and clause (6) as well as the relationship among clauses (8), (9), (10) and (11) is parallel progression because the same topical subject is used in the consecutive clauses. As a matter of fact, unless there is no such topical subject "some" in clause numbers (7), clauses from number (5) to number (11) constitute the global parallel progression because the topical subjects in the latter clauses refer back to the former clauses. In other words, clauses from number (5) to number (11) form the extended parallel progression due to the fact that there is one clause with different topical subjects between the first mention of the topic in clause number (5) and its recurrence in clause number (8). Another parallel progression which is much more covert is between clause (2) and the clause (22), with so many clauses of different topical subjects in between. The theme "one such American expression" alters into the theme "the expression" in clause number (22); referring to the identical topical subject though the two linguistic expressions are a little bit different.

Sequential Progression: The comment in clause number (1) "words and expressions" becomes part of the topic of the next clause number (2), viz. "one such American expression". And again the comment "eager beaver" in clause number (2) turns into the topic of the following clause number (3), viz. "an eager beaver" .This is the typical exemplification of the Sequential Progression in TSP or the Simple Linear Progression involved in the Hu's Patterns of thematic progression. In the case of clause number (15) and (16), they also fall into the category of Sequential Progression.

Extended Sequential Progression: The comment "the beaver" in clause number (18) changes into the topic of clause number (21) "wild beavers", inserting two clauses with different topical subjects between them. This conforms to the definition of the extended sequential progression: the comment element of a clause is taken up as the topic of a 
non-consecutive clause.

The article explains the origin of the American idiomatic expression "eager-beaver". The "Expression" is the major content and the ultimate purpose that the writer desired to convey. To drive home this point, "expression" itself is used three times in the article. From theme 5 to theme 10, the writer reveals the eager-beaver's character step by step. He changes the subject from theme13 and expresses the effect of the eager-beaver in history. The theme returned to "expression" at the end of the discourse. The whole discourse is complete and coherent because it works in concern with the front and the back. Hence, we can conclude that this piece of expository discourse achieves unity and coherence with the smooth progression of the theme.

\section{B. The Thematic Progression in a Narrative Text}

This piece of chosen discourse "a private conversation" is a rather classic and typical narration popular both in some English course books and via the Internet webpage. A lot of discourse analysts utilize this to expound the Labov Model, an analytic model of narrative. To verify the thematic progression models explicated in the first section of the present paper, the author carries out a discourse analysis of this well-known narrative text through considering the pattern of thematic progression. For the sake of conformity, the writer employs the same symbols as above to notify the different sentence elements: Italics are used for ISE, underlining is employed to signal mood subject (grammatical subject of the verb), and bold face is for the topical subject.

(1) Last week $\underline{I}$ went to the theatre. (2)I had a very good seat. (3) $\underline{\text { The }}$ play was very interesting. (4) $\underline{I}$ did not enjoy it. (5)A young man and a young woman were sitting behind me. (6) They were talking loudly. (7) $\underline{I}$ got very angry. (8) $\underline{I}$ could not hear the actors. (9) $\underline{I}$ turned round. (10) $\underline{I}$ looked at the man and the woman angrily. (11) $\underline{T h e y}$ did not pay any attention.(12) In the end, $\underline{\mathbf{I}}$ could not bear it. (13) $\underline{I}$ turned round again. 'I can't hear a word!' I said angrily. (14) 'It's none of your business,' the young man said rudely.' This is a private conversation!'

Parallel Progression: In the clause number (5), "a young man and a young woman" acts as the ISE, the mood subject and the topical subject. And the pronoun "they" in the clause number (6) embodies the three sentence elements at the same time and refers back to the young man and young woman. Therefore, the thematic progression here is a parallel one. Clauses (7), (8), (9) and (10) are obviously the parallel progression since they all share the same topical subject, playing the part of the three sentence elements concurrently, which all stand for the author oneself.

Extended Parallel Progression: The Pronoun "I" in clause number (2) and The Pronoun "I" in clause number (4) co-refer to the author ,intervened by the topical subject "The play" of the clause number (3) in between, which interrupts the continuous progression of the theme from clause (2) to clause (4). Such strategy of topical progression is defined as the extended parallel progression. In the same way, the topical subject "they" in clause (11) breaks the chain of the topical progression among clauses (7), (8), (9), (10) and clauses (12) and (13). This way of topical progression can be classified into the extended parallel progression.

Sequential Progression: In this short piece of discourse, there exists only one sequential progression. The comment element "the man and the woman" changes into the topic of the approaching clause, despite the truth that the different linguistic expressions are used to signify the same entity within the discourse.

Extended Sequential Progression: The topical subject "me" in the rheme part of clause (5) turns into the theme of clause (7), between which lies in the clause (6) with a different topical subject.

The above analysis goes completely well with the TSP of Lautimatti and Simpson. Apart from the four kinds of topical progression in this piece of discourse, we can unveil another kind of progression mode which is not mentioned in TSP but in the Main Patterns of the thematic progression patterns proposed by $\mathrm{Hu}$. It is the Converse Linear Progression that is identical with the cross pattern proposed by Shenghuan Xu (1982), which is why this piece of text is coherently organized. The converse Linear Progression can be diagramed as follows: (refer to Hu, P93)

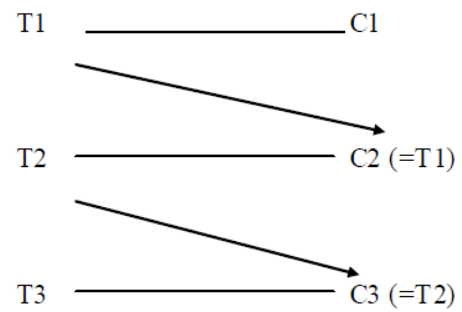

Converse Linear Progression: The topic "the play" in clause number (3) turns into the comment element "it" in clause (4), which has never been mentioned by the TSP model.

Up to now, borrowing from the experience of Simpson's revision to the Lautimatti's three types of TSP, the author preliminarily attempts to make the addition of another type of the topical progression---the extended converse linear progression, which is of much great necessity in analyzing the present text. The so-called extended converse linear progression can be defined as follows: the topic of the previous clause becomes the comment element of the latter clause between which there stand many other clauses, which is labeled as follows: 


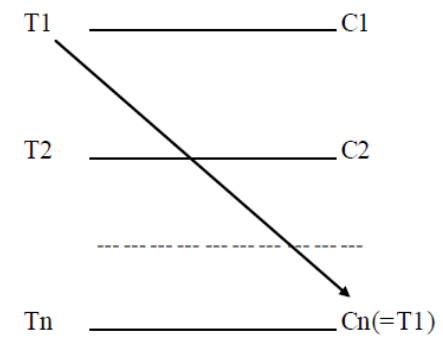

Now let's apply this new model to the analysis of the narrative discourse.

Extended Converse Linear Progression: The topic "a young man and a young woman" in clause number (5) changes into the comment element "the man and the woman" in clause number (10), with clauses (6), (7), (8) and (9) intervened in between.

\section{RESULTS AND DisCUSSION}

\section{A. Enlightenment from the Combination between TSP and Hu's Patterns of TP}

Based on Lautimatti's three types of TSP, Simpson proposed that it was essential to put forward the fourth type of TSP, namely the extended sequential progression. The author's analysis of the first piece of discourse in the present article utterly follows the four models and thoroughly understand the internal coherence and cohesion .when it comes to the second text, a much familiar one, the paper finds that only the four types of TSP can not satisfy the needs of the explication of the topical progression so that it advises that we should combine the Converse Linear Progression in the Hu's TP Pattern with the original four types of topical progression in TSP(the reason has been expounded in the comparison of the two models of theory in the section of literature review of the paper) and accordingly finds out the corresponding examples to prove this. Furthermore, while carefully analyzing the topical progression in the second text, the author thinks that the already mentioned five types TSP can not totally meet the requirements of the analyzing the second piece of discourse. Therefore, the author here tries to advance the sixth type of topical progression -the extended converse linear progression to perfect the incorporation.

The present paper has done two innovative things: firstly, through the comparison and contrast of Simpson's(2000) new models of TSP and the main patterns of TP held by Yinglin Hu (2006), the author proposes that we should build a new model of TSP by means of incorporation of the two models. Secondly, during the course of analysis by way of the newly incorporated model, it is reflected that the five types of TSP are still not enough to interpret the topic progression models in such a classic narration. Hence, the author makes a tentative attempt to put forward the sixth type of TSP, viz. the extended converse linear progression.

How the themes relate to other themes and rhemes in a well-organized text is much more diversified and most linguists only recommend some primary progression models. A discourse can reach cohesion and coherence in various ways by setting up relations between and among the themes and rhemes of the clauses or between and among the topics and comments of the sentences, adopting more than one progression model. This paper, on the ground of combining the major modes of topical progression submitted by Simpson and Yinglin Hu, discovers two new TSP models: Converse Linear Progression and Extended Converse Linear Progression.

Jian Shi (2009) confused the concepts differences between theme and rheme as well as topic and comment and deduced some ambiguous progression modals, so this paper, clarifying such basic conceptions firstly, takes preference to Simpson's TSP modal combining itself with Yingling Hu's TP patterns and revised the hypothesized TSP modals with C replacing R(C Signifying Comment while R denoting Rheme), T referring to Topic instead of Theme.

\section{B. The Limitations of the Study}

The author tries to enrich the models of topical structure progression based on the theoretical comparison between the Simpson's TSP model and the TP Patterns maintained by Yinglin Hu as well as the practical analysis of the popular narrative discourse "a private conversation". There is not shortage of appropriateness for us to put the two models together after comparing and contrasting their strong points and weaknesses respectively. The real problem lies in the fact that in the present article, the author merely locates the uniqueness of the thematic progression in one part of the available discourse although it is a well-known one, which lacks adequate evidence to prove its acceptability. What is in great need is that we should not only test the newly suggested six types of TSP in a large quantities of narratives, but in the vast amount of other genres of discourse such as expository writing, argumentative writing as well. Due to the limitations of time and space of the present article, we will discuss these questions and testify the hypothesized model of TSP in other articles.

\section{REFERENCES}

[1] Bing Li (2012). An Experimental Study of Coherence of Argumentative Writings from the Perspectives of Genre Theory and Thematic Progression Theory, Jinan: Shan Dong University. 
[2] Danes, F. (1974). Functional Sentence Perspective and the Organization of theText, in F. Danes(ed.). Papers in Functional Sentence Perspective. Prague:Academia, 106-128.

[3] Fries, P. H. (1983). On the Status of Theme in English, in Janos S.Petofi and Emel Sozer(ed.). Micro-and Macro-connexity of Discourse. Hamburg: Buske, 116-152.

[4] Fries, P. H. (1995). Theme, Methods of Development and Texts, in R. Hasan and P. Fries (ed.): On Subject and Theme: A Discourse Functional Perspective . Amsterdan: John Benjamins, 1995. 317-359.

[5] Guoqing Li. (2003). TP Modal and Textual Genres: Analysis of Old Man and Sea, Foreign Languages and Their Teaching 2003(7) 53-56.

[6] http://school.ecp.com.cn/resource/UploadFiles/2006715114129553.doc (retrieval time: 2006/7/15).

[7] http://insuns.com/english/vision/photo/20061003/720.html (retrieval time: 2006/7/15).

[8] Huisong Zhang. (2005). The Thematic Progression and Topic Progression in Texts (1) Journal of Hubei Normal University (Philosophy and Social Science) 2005(3), 64-68.

[9] Huisong Zhang. (2006). The Thematic Progression and Topic Progression in Texts (2) Journal of Hubei Normal University (Philosophy and Social Science) 2006(6), 101-103.

[10] Jian Shi. (2009). The Discussion of TSP Modals in English Texts, Tokyo Literature 2009(4) 106-108.

[11] Lan Xiang. (2002) The Application of TP Modals in the Teaching of Reading, Foreign Languages and Their Teaching 2002(3) 61-63.

[12] Lautimatti, L. (1987). Observations on the Development of the Topic in Simplified Discourse. Helsinki: Akateemineen Kirjakawppa.

[13] Martin,J. R. (1992). Theme, Method of Development and Existentiality: The price of reply. Occasional Papers in Systemic Linguistics.

[14] Mei Chen. (2005). Theme-Rheme Theory and the Textual Coherence in College English, Writing Shandong Foreign Language Teaching Journal 2005(6) 70-73.

[15] Michael McCarthy. (1991). Discourse Analysis for Language Teachers, Cambridge: Cambridge University Press.

[16] Qi Wang. Xiaotang Cheng. (2004) Thematic Progression and Information Parameters in Texts, Foreign Language Research, 2004(2), 48-52.

[17] Simpson. (2000). Topical Structure Analysis of Academic Paragraphs in English and Spainish, Journal of Second Language Writing, 9(3), 293-309(2000).

[18] Shenghuan Xu. (1982). On Theme and Rheme, Foreign Language Teaching and Research, 1982(1), 1-9.

[19] Shenghuan Xu. (1985). On Theme and Rheme Again, Foreign Language Teaching and Research, 1985(4), 19-25.

[20] Xuanwei Peng. (2005). System of Topic Chain in Discourse, Foreign Languages Research 2005(4) 6-13.

[21] Yan Liu. (2010). On Thematic Progression, Xi'an Social Science 2010(8) 134-136.

[22] Yan Huang. (1985). On the English Theme and Rheme, Journal of Foreign Languages 1985(5) 32-36.

[23] Yinglin Hu. (2006). Discourse Analysis Wuhan: Central China Normal University Publishing House P 90-93.

Jian Shi was born in Shaanxi, China in 1977. He received his master's degree in Foreign Linguistics and Applied Linguistics from the University of Shaanxi Normal University in 2010.

$\mathrm{He}$ is currently a lecturer in College of Humanities and Foreign Languages, Xi'an University of Science and Technology, China. His major research interests include English Education, Second Language Education, Discourse Analysis and Cognitive Linguistics. 